Digital Press Social Sciences and Humanities

L'image de la marque française dans les cosmétiques coréennes

Dian Agustina Pratama, Silvi Satiakemala and Agnes Andryani Rosiana

Proceeding of Conférence internationale sur le français 2018

Joesana Tjahjani, Merry Andriani, Sajarwa, Wening Udasmoro (eds) 


\title{
L'image de la marque française dans les cosmétiques coréennes
}

\author{
Dian Agustina Pratama*, Silvi Satiakemala , et Agnes Andryani Rosiana
}

STBA YAPARI Bandung,Bandung, Indonesia

*e-mail : districtde@gmail.com

\section{Résumé}

De nos jours, l'industrie cosmétique se développe bien rapidement. Des nombreuses inventions se produisent surtout en France, mais aussi dans d'autres parties du monde comme en Corée du Sud. Il est indéniable que la Corée du Sud étant le 10ème marché mondial, produit des variétés de marques cosmétiques connues dans le monde. La beauté routine célèbre « 10 étapes de soins de la peau » est introduite par ce pays et devenue la nouvelle inspiration parmi les femmes. Cette étude s'intéresse spécifiquement à l'identité de la marque française dans les cosmétiques coréens. En effet, la marque d'une cosmétique est vraiment pertinente afin de promouvoir son image auprès des partenaires et des clients. Elle associe également à la culture d'un pays d'origine. La France comme un pays ayant le grand marché de produits de beauté influence la sélection des marques dans des cosmétiques coréennes. Quelques produits de beauté coréens utilisent le français dans leurs marques. Il est considéré que la supériorité et l'image hédonique de la langue française peut améliorer la valeur d'une marque cosmétique. Ces marques expriment également la similarité entre la Corée du Sud et la France, le concept des cosmétiques naturelles.

\section{Mots-clés}

marque, français, cosmétique, coréen, identité, foreign branding

\begin{abstract}
Nowadays, the cosmetic industry is developing very quickly. Many inventions occur mainly in France, but also in other parts of the world as in South Korea. It is undeniable that South Korea being the 10th largest market in the world produces varieties of cosmetic brands known worldwide. The beauty routine that is called "10 steps of skin care" is introduced by this country and become the new inspiration among women. This study focuses specifically on the image of the French brand in Korean cosmetics. Indeed, the brand of a cosmetic is really relevant to promote its image with partners and customers. It also associates with the culture of a country of origin. France as a country with large beauty products market influences the selection of brands in Korean cosmetics. Some Korean beauty products use French in their brands. It is considered that the superiority of the French language can enhance the value of a cosmetic brand. These brands also express the similarity between South Korea and France, the concept of natural cosmetics.
\end{abstract}

\section{Keywords}

brand, French, cosmetic, Korean, identity, foreign branding

\section{Introduction}

Grace à ses produits cosmétiques, La Corée du Sud est devenue l'un des pays les plus célèbres dans le monde de beauté. Aujourd'hui, les consommateurs préfèrent plutôt les cosmétiques coréens que ceux du Japon qui sont premièrement très connus au monde entier. Les bloggeuses et vloggers de beauté dans des parties du monde cherchent toujours des nouvelles informations sur les cosmétiques, les produits même 
les méthodes de maquillage appliquées par les coréens. Ceci est influencé par le développement de la culture sud-coréenne qui s'est répandue dans de nombreux pays. Ce phénomène a eu un impact positif dans le domaine de la mode et de la beauté. Les visages des actrices et des acteurs sud-coréens ou des membres de groupes musicaux semblent toujours naturels, propres, sains, clairs et lisses, faisant que les hommes et les femmes veulent avoir des visages comme eux.

Le concept de cosmétique porté par la Corée du Sud est différent des cosmétiques occidentaux. Une autre particularité, les marques coréennes mettent en avant divers produits de soins du visage à base naturelle. Les produits cosmétiques coréens ont plus de types que les cosmétiques en général, de sorte que la Corée est également considérée comme plus innovante dans la fabrication de produits cosmétiques, l'une d'elles étant la crème $\mathrm{BB}$, la crème $\mathrm{CC}$. Les cosmétiques sont multifonctionnels car ils ne contiennent que la crème hydratante mais aussi l'écran solaire dans un produit de maquillage. La routine de soins de la peau coréens est une référence pour les femmes pour obtenir des résultats éclatants, propres et frais. Une routine de soins de la peau coréenne en 10 étapes, une peau de verre et diverses méthodes de beauté en provenance de Corée sont ciblées pour que le visage ressemble à une femme coréenne. Depuis le boom avec K-Waves, de nombreux produits coréens ont été exportés en Europe et en Amérique.

La France est un pays souvent connu comme le centre des cosmétiques et le premier exportateur mondial. Elle est le premier pays qui a importé K-Beauty en 2015. Les Français profitent d'une gamme de produits cosmétiques coréens de grande qualité à des prix moins élevés que les produits cosmétiques français. Après tout, les cosmétiques coréens sont plus souvent fabriqués à partir d'ingrédients naturels afin de rendre le maquillage et le visage plus naturels. Les cosmétiques français qui ont une image de marque avec l'impression de luxe et de coût font de la France une référence pour les tendances de la beauté. L'image du pays français affecte la dénomination de marques de produits cosmétiques dans différents pays utilisant le français, une autre raison de l'utilisation du français en tant que marque cosmétique est considérée comme plus acceptable et peut augmenter la valeur du produit. La décision de marque est une étape très importante qui représente l'objectif d'un produit cosmétique et de son segment de consommation. L'un d'eux est la Corée du Sud avec les marques Laneige, eSpoir, Mamonde, Staging, Verity et lacvert. La philosophie de l'utilisation du français en tant que marque de produits cosmétiques est visible sur leur site officiel (Dauk \& van Zomeren, 2018). Cette étude se concentre sur les marques de cosmétiques coréennes qui utilisent le français et la philosophie et également l'identité de marque que les entreprises espèrent de ses consommateurs.

\section{Discussion}

\subsection{Les marques françaises dans les cosmétiques coréennes}

Le français peut être trouvé dans les marques de mode, de gastronomie, de parfum et de cosmétiques. L'utilisation du français dans de nombreuses marques est indissociable de l'image du pays (France) dans ce domaine. La France est le plus grand exportateur de cosmétiques au monde. Il a des marques populaires qui ont la valeur de lux par rapport au produit d'autres pays. Le français est également largement utilisé comme terminologie ou information sur les emballages de produits cosmétiques, par exemple le baume pour les lèvres, le rouge à lèvres, le fond de teint, la poudre libre, etc. Donc, la Corée fait la même chose. Il profite du français comme marque pour augmenter la valeur marchande du produit.

Les marques coréennes qui utilisent le français comme marque sont La neige, eSpoir, Mamonde, Miseen-scène, Verite et Lacvert. Selon Leclerc (1994, p. 264), ces marques françaises ont des significations plus positives que la marque anglaise. Il a l'image hédonique, le luxe et a une prononciation intéressante qui est plus acceptée que les marques dans d'autres langues. Cette valeur hédonique a une association de joie que l'on utilise ces cosmétiques (Batra \& Athola dans Mobarec \& Friedmann, 2009, p. 13).

Presque tous les cosmétiques coréens ont le concept naturel et organique avec des matériaux souvent utilisés par les Asiatiques. Ce concept de soins du visage est offert par une prévention saine pour la peau, claire et anti-âge. Ensuite, pour le maquillage, le concept utilisé est naturel et pas aussi épais que le maquillage américain. Les cosmétiques coréens connus sous le nom d'innovations, par exemple la crème matelassant, la teinte pour les lèvres, le fondant (fond de teint), le baume trouvé (baume avec fond de teint), etc.

Sur les six marques de cosmétiques coréennes trouvées, presque tous les Coréens peuvent les prononcer. La marque doit être facile à prononcer par le consommateur de différents pays. Les mots français choisis pour les marques de cosmétiques sont acceptables pour les Coréens. Quand on entend ou 
écrit une marque française, le sujet peut être axé sur la qualité de la beauté française et conclu sur la qualité du produit hédonique (Leclerc et al., 1994, p. 268). La sélection de la marque française doit être adaptée à la vision et à la mission de l'entreprise.

La France a contribué à façonner la perception des consommateurs sur les produits cosmétiques en provenance de Corée du Sud. Ce sont les Français qui se soucient de leur peau dès leur plus jeune âge, l'existence de nombreuses pharmacies en France est la prouve. Le concept de la beauté française est montré naturellement ainsi que le concept de la beauté en Corée du Sud. La sélection de marques français par des entreprises non françaises concerne également les consommateurs. Peabody (dans Olavarrieta et al., 2009, p. 13) a expliqué que les stéréotypes culturels peuvent renforcer l'effet de l'utilisation de marques en langue étrangère. Dans ce cas, il ne peut pas être nié que la France a un stéréotype d'avoir un bon goût et un haut niveau de beauté. Laneige, Mamonde, Lacvert et Verité ont des significations naturelles et littérales, en ligne avec les marques d'histoire des sociétés de cosmétiques coréennes. Les quatre produits sud-coréens sont une série de soins de la peau visant à soigner la santé du visage, tandis que eSpoir, Mise-en-scène et A'Pieu signifient à pouvoir avoir une apparence parfaite. Cela peut être vu à partir des produits publiés, qui sont des produits de maquillage et de cheveux.

Les produits cosmétiques Sud-Coréens en français sont la bonne étape pour placer la Corée du Sud au centre des tendances cosmétiques autres que la France elle-même. La Corée du Sud a un ensemble plus complexe de produits cosmétiques et est devenue sa propre tendance. L'utilisation du français pour les produits de beauté coréens permet à leurs produits d'être plus facilement reconnus et recherchés sur le marché international. Un autre avantage est que le français a une écriture latine plus facile à lire. Les lecteurs de Laneige, Mamonde, Verité, Lacvert, eSpoir, et Mise-en-scène ne rencontrent pas de difficultés cruciales lorsqu'ils sont lus par des personnes ne parlant pas français.

En Indonésie, Laneige et Mamonde sont connus comme des produits cosmétiques en provenance de Corée du Sud. Quatre autres produits ne sont pas trop pris en compte par les blogueurs de beauté ou les vloggers indonésiens. En règle générale, Verité, Lacvert, eSpoir, et Mise-en-scène seront considérés comme des produits français et pas des produits sud-coréens si nous ne recherchons pas d'autres informations sur l'origine du producteur. De telles erreurs sont fréquentes lors de la lecture de marques de langues étrangères. Les consommateurs associent généralement une marque de produit en tant que produit authentique du pays de la langue utilisée.

\subsection{L'identité de la marque française}

La marque est une chose très importante pour un produit commercial, y compris les produits cosmétiques. C'est très important et cela peut influencer les consommateurs. Elle est comme l'identité inséparable d'un produit. Comment l'entreprise donne-t-elle l'image sur le produit cosmétique sur le marché, nous pouvons savoir par la marque. Ceci est renforcé par Roy et Banerjee (2007) qui ont déclaré qu'une marque aide le client à distinguer un produit d'un autre. Souvent, nous pouvons connaître le standard d'un cosmétique par la marque.

Roy et Banerjee (2007), ils ont également ajouté que l'identité de marque peut être constituée de traits de caractère, d'avantages, de valeurs, de différenciation et de personnalité. Cela peut être vu comme tout ce que le propriétaire de la marque veut que les consommateurs associent à la marque. A cause de ça l'entreprise doit donner la marque attirante pour que les consommateurs s'intéressent à l'acheter. Ainsi l'identité de marque est le sens de la marque, ce qui donne un sens et ce qui la rend unique; c'est une empreinte de marque. Il est autant expliqué par Rosengren, Standoft, et Sundbrandt (2010) qu'il existe deux sorts de l'identité de la marque : l'identité et l'identité prolongée.

En plus, l'identité essentielle est l'âme de marque et elle doit se faire des choses principales qui font les marques précieuses et originales. L'identité essentielle de marque doit être solide, stable et ne pas changer de temps en temps, indépendamment des changements de positionnement de la marque et des stratégies de communication (Rosengren et al, 2010, p. 5). De l'autre côté, l'identité étendue peut changer pour des fins de marketing. La valeur ne peut pas être trop forte et fondamentale.

Ces marques françaises trouvées ont également un caractère unique tel que décrit dans leurs produits cosmétiques. La singularité tirée du sens de la marque devient l'identité de chaque marque et se maintient même si les produits cosmétiques fabriqués ont de nouvelles innovations mais ne peuvent être séparés de l'histoire de la marque qui a été construite. Buffer (à Jeconiah, 2015) diviser l'identité de marque en six dimensions ;

1. La Physique, c'est une caractéristique va bientôt se former dans la pensée quand nous pensons de la marque. 
2. La Personnalité, décrit qu'on va devenir dans quel humain avec quelle caractère physique quand une marque est assimilée à l'humain.

3. La culture comme un ensemble de valeurs qui sont l'inspiration d'une marque.

4. La relation, chaque marque est obligée de maintenir de bonnes relations avec les clients.

5. La réflexion est une description de ce que les consommateurs veulent être vus lorsqu'ils utilisent une marque.

6. L'image de soi est un reflet interne de la cible du consommateur.

Dans cette étude, les marques de cosmétiques qui utilisent le français répondent aux dimensions de la personnalité et de la culture. Les dimensions de la personnalité ne sont pas présentées sous une forme humaine, mais dans la nature, l'abstraction et le monde, en accord avec le sens littéral du français, tandis que la dimension de la culture est incorporée dans la valeur philosophique de la marque.

La marque Laneige a le sens «neige » et écrit sur son site web officiel que la philosophie de ce produit est de rendre la peau plus blanche que la neige, pour que les femmes se sentent plus en confiance avec leur beauté intérieure. Et chaque flocon de neige est différent et unique, comme chaque femme. La marque a pris ce nom pour encourager chaque femme à mettre en valeur la beauté qui s'y trouve. Laneige a un slogan sparkling beauty « la beauté pétillante » qui est compatible avec ses produits basés de l'eau pour blanchir la peau comme la neige. Ce caractère deLaneige est connu pour son identité, malgré les nombreuses innovations.

Mamonde vient des deux mots français ma et monde, grammaticalement ce sont incorrect, il faut utiliser le terme mon et monde parce que le mot monde est masculin. Et pour la raison de la prononciation, on simplifie comme mon monde. Les produits Mamonde sont reconnus par des matériaux des toutes les parties des fleurs. Le slogan est inspiré par des fleurs. Les fleurs sont identifiées au féminin et au femmes, les produits cosmétiques visent donc à comprendre le monde des femmes, allant du soin de la peau, du maquillage et d'autres produits de soins. La beauté des fleurs vient aussi de la nature, de la terre, de la lumière du soleil, du vent et de l'eau.

eSpoir en français est le sentiment qui conduit à espérer. Cette marque croit au pouvoir de la transformation que le maquillage peut nous rendre plus belle et confidente. Ce produit donne l'espoir aux femmes de changer comme elles veulent. Le slogan de cette marque est le pouvoir de transformation. Les produits cosmétiques qui sont délivrés ont également des couleurs de maquillage plus larges et prétendent avoir une solution pour tous les problèmes de maquillage de base. Comme son nom l'indique, cette marque est en effet un espoir pour les femmes d'exprimer leur beauté originale.

Verité en française a le sens « la vérité » ou « la réalité ». Ce produit vise à trouver et à fournir des solutions réelles aux traitements du visage. Son identité de marque est versée dans des produits de soins de la peau qui réduisent les rides, évitent la sécheresse du visage et éclaircissent la peau du visage pour qu'elle apparaisse comme la « vérité » du vrai visage. Lacvert en française vient des mots lac et vert. Il a la philosophie de fournir des solutions pour les soins du visage sensitive, et à base de formule naturelle.

Lacvert vient de deux mots français « lac » et « vert». Il signifie un lac qui est vert. Cette philosophie de marque vise à aider la peau à éviter le dessèchement. Cette marque contient une série de produits cosmétiques qui hydrate et nourrit la peau. Comme le nom de la marque, lac a le caractère naturel, alors il espère que par son produit qui est naturel pourra être la solution pour la peau sensible.

Mise-en-scène utilise le terme " direction » d'un film pour produire des cheveux sains et faciles à agencer en fonction de la coiffure désirée. Cette identité de marque peut être vue à partir de la valeur et du caractère des produits de soins capillaires délivrés. L'esthétique du film apparaît dans les produits de coloration Mise-en-scène et les produits de soins capillaires pour la santé des cheveux.

\section{Conclusion}

Les dimensions de l'identité de marque française dans la cosmétique coréenne qu'on a trouvé dans cette étude sont l'identité de la personnalité et de la culture. La personnalité, c'est la facette interne de la marque française. Ces marques françaises trouvée montrent la caractéristique de la nature, de l'abstrait, et de la mondanité. Et aussi, la dimension culturelle montre la valeur de marque et participe des cultures cosmétiques coréenne et française. Les entreprises cosmétiques coréennes utilisent les ingrédients naturels, c'est l'un des raisons de choisir la marque cosmétique en français. L'autre, les marques françaises donnent l'image hédonique et luxe qu'on espère que ca pourra augmenter la valeur de produit. 


\section{Références}

Dauk, B., \& van Zomeren, E. (2018). Brand Names and the Use of Hedonic Symbolism. In Student Research Symposium. Salt Lake City: Utah State University. Retrieved from https://digitalcommons.usu.edu/cgi/viewcontent.cgi?article=1664\&context=researchweek Jeconiah, L. (2015). Analisa Pengaruh Brand Identity terhadap Pembentukan Brand Image dengan Promotion Mix dan Brand Awareness sebagai Variabel Intervening pada Merk SPEEDO. Jurnal Manajemen Pemasaran, 3(1), 1-11. Retrieved from http://publication.petra.ac.id/index.php/manajemen-pemasaran/article/view/4275

Leclerc, F., Schmitt, B. H., \& Dubé, L. (1994). Foreign Branding and Its Effects on Product Perceptions and Attitudes. Journal of Marketing Research, 31(2), 263-270. https://doi.org/10.2307/3152198

Leclerc, F., Schmitt, B. H., \& Dube-Rioux, L. (1989). Brand Name a La Francaise ? Oui, But For the Right Product! Advances in Consumer Research, 16, 253-257. Retrieved from http://acrwebsite.org/volumes/6911/volumes/v16/NA-16

Olavarrieta Soto, S., Manzur Mobarrec, E., \& Friedmann, R. (2009). Foreign Branding: Examining the Relationship between Language and International Brand Evaluations. Innovar, 19(35), 9-18. Retrieved from http://www.scielo.org.co/pdf/inno/v19n35/19n35a02.pdf

Rosengren, A., Standoft, A., \& Sundbrandt, A. (2010). Brand Identity and Brand Image A Case Study of Apotek Hjärtat. Jönköping University. Retrieved from http://www.divaportal.org/smash/get/diva2:326094/fulltext01.pdf

Roy, D., \& Banerjee, S. (2007). CARE-ing strategy for integration of brand identity with brand image. International Journal of Commerce and Management, 17(1/2), 140-148. https://doi.org/10.1108/10569210710776512 